\title{
Mensaje e interacción en el ensayo de crítica literaria en lengua inglesa. Análisis del título y de la introducción ${ }^{1}$
}

\section{Emilia RÉBORA TOGNO \\ Universidad Nacional Autónoma de México}

El estudio del discurso es sin lugar a dudas un campo difícil de definir. Podemos partir del discurso, valga la redundancia, y explicarlo como un suceso de comunicación que implica aspectos funcionales tales como quién utiliza el discurso, cómo, por qué y cuándo lo hace.

En el campo de dicho estudio identificamos tres dimensiones: a) el uso de la lengua, b) la comunicación o transmisión de algo (ideas, hechos, sentimientos, etcétera) y c) la interacción entre participantes. Al referirnos al discurso como un suceso de comunicación, no nos limitamos a la conversación, i.e. un discurso de carácter oral y visual, sino que consideramos asimismo al texto escrito.

Estas tres dimensiones explican la participación de varias disciplinas, que a manera de herramientas apoyan el análisis del discurso: la linguística, la psicología y las ciencias sociales. Podemos representar el campo en cuestión como un triángulo, cuyos vértices son la estructuración del texto y de la conversación, la cognición y la estructura social o cultura. Cada uno de los vértices de este triángulo puede convertirse en el terreno focal del análisis en sí, pero la relación entre los vértices obliga al estudioso a recorrer los segmentos que los unen. El análisis del discurso es por lo tanto un campo transdisciplinario.

La vastedad y complejidad del campo obliga a la especialización; los estudiosos se ven en la necesidad de delimitar su objeto de estudio pero sin perder de vista la naturaleza social, contextual, interactiva y funcional que lo conforma.

El estudio que aquí presento se ubica dentro de estos parámetros burdamente trazados. Abordo el análisis del título y de la introducción de cinco ensayos de crítica literaria a partir de un enfoque funcional. ${ }^{2}$

${ }^{1}$ El presente trabajo es una versión sucinta de la tesis que para obtener el grado de Maestría en Linguística Aplicada presentó la autora.

${ }^{2}$ Lyons (1981: 224-228) sitúa el funcionalismo en lingüística como una corriente del estructuralismo, cuya tesis fundamental es que la estructura de la lengua está deter- 
Desde una perspectiva funcional, el ensayo, igual que cualquier texto, responde a una situación, a ciertos objetivos establecidos culturalmente, socialmente. En términos generales, podemos decir que el ensayo transmite ideas: propone, refuta o bien apoya una tesis; tiene un carácter exploratorio, polémico y didáctico. Souto (1979) nos dice que existe una condición que todo ensayo debe cumplir y ésta es la claridad de expresión, una transparencia que puede darse de diversas maneras. El contenido o mensaje se realiza a través de un diálogo entre el escritor y el lector. El ensayo, por lo tanto, tiene una función doble: interactiva/social y transmisora del conocimiento. De acuerdo a la tesis fundamental del funcionalismo, dichas funciones se manifiestan en el texto.

En cuanto a su organización, el ensayo, canónicamente, contiene un título, una introducción, un cuerpo y una conclusión.

El estudio que aquí.se presenta incursiona en el análisis de la doble función del ensayo de crítica literaria, a través de categorías sintáctico-funcionales, en la introducción y el título. Parto de la premisa que la introducción, el título y la conclusión sirven para abrir y cerrar el canal de comunicación entre el escritor y el lector, así como para plantear el contenido. Por lo tanto, considero que en estas secciones será especialmente evidente la doble función del ensayo. El presente trabajo sólo aborda el análisis de la introducción y el título.

Las categorías sintáctico-funcionales que se analizarán son: la configuración de la introducción de los ensayos, en cuanto a su organización temática a partir del análisis de las cláusulas finitas y, la relación entre la configuración temática con el título del texto complementado con el análisis de la estructura de la información (dado-nuevo).

El marco teórico que sustenta el estudio reúne propuestas de varios lingüistas dentro del enfoque funcional: Halliday, Hasan, Brown y Yule, y Daneš.

Halliday se sitúa dentro de la tradición funcional europea. Sus raíces las encontramos en la Escuela de Londres y en los principios del Círculo de Praga. El concepto básico del que parte es el sistema en el sentido de Firth:

minada por las funciones que desempeña en la sociedad en la que opera. A esta corriente pertenecen el Círculo de Praga, el Círculo de Copenhague y la Escuela de Londres, entre otros. Aun cuando existen marcadas diferencias entre las escuela funcionalistas, todas comparten el principio de la multifuncionalidad de la lengua como instrumento de comunicación. Conciben la lengua como una red de relaciones y las estructuras como su realización. El uso y el contexto comunicativo determinan la estructura de los enunciados. El objeto de estudio del funcionalismo lingüístico es el texto y el sistema: el segundo es necesario para comprender al texto y éste es la razón última para el estudio del sistema. 
un paradigma funcional desarrollado en un constructo formal, una gama de posibilidades y condiciones de entrada, de selección, "A formal paradigm... developed into the formal construct of a 'system network" (Halliday 1985: xxvii).

Halliday interpreta la lengua dentro de un contexto sociocultural, en el que la cultura se define en términos semióticos (el campo, el tenor y el modo). A través de metafunciones (ideacional, interpersonal y textual), Halliday vincula la semiótica con el texto. Halliday en An Introduction to Functional Grammar (1985) ofrece una gramática descriptiva, funcional y semántica; una gramática que permite describir al texto en términos formales y explícitos pero relacionándolo con el entorno situacional y cultural a través de categorías semánticas. En esta gramática, las categorías sintácticas son consideradas como realizaciones de categorías semánticas.

Debido a que el modelo sistémico-funcional de Halliday y su gramática son la columna vertebral del marco teórico que sustenta el estudio, abundaré sobre el mismo.

De una manera muy general, el modelo sistémico-funcional de Halliday ve los componentes del lenguaje (semántica, morfosintaxis y fonología) como un potencial de alternativas, una red de posibles relaciones paradigmáticas. El sistema semántico está dentro y fuera del lenguaje y constituye un potencial de comportamiento; es parte de la semiótica social que se proyecta en el sistema lingüístico. Para Halliday:

El texto es la forma lingüística de interacción social; es una progresión continua de significados, que se combinan tanto simultáneamente como en sucesión. Los significados son la selección hecha por el hablante entre las opciones que constituyen el potencial de significado; el texto es la realización de ese potencial de significado, el proceso de elección semántico (Halliday 1982: 160).

La situación no se lleva a cabo en abstracto sino dentro de una situación particular, específica. La situación es el entorno en que el texto aparece. La estructura social define y da valor a los diversos tipos de contexto social en que se intercambian los significados, en que se crean textos. La estructura semiótica de la situación está constituida por tres variables: el campo, el tenor y el modo. El campo es el asunto a tratar; el tenor es la acción social en que se inserta el texto, las opciones interpersonales y discursivas de los participantes; el modo se refiere a los canales que se adoptan, el conducto retórico con sus estrategias asociadas. El campo, el tenor y el modo no son tipos de uso del lenguaje sino un marco conceptual que permite la explicación del contexto social. 
Entre el contexto social y las estructuras lingüísticas se encuentra la noción de registro, la cual se refiere al hecho que el lenguaje que hablamos varía de acuerdo con el tipo de situación. El registro está determinado por las variaciones espacio-semióticas del campo, tenor y modo, las cuales actúan no solamente sobre la situación, sino a través de la semántica en el sistema lingüístico.

El sistema semántico es un entrefase entre el sistema lingüístico y el contexto social. Los componentes funcionales de la semántica desempeñan el papel de metafunciones, formando la base de la organización del sistema lingüístico; el campo se realiza a través de la metafunción ideacional, el tenor a través de la metafunción interpersonal o modal y, el modo a través de la textual. ${ }^{3}$

Cada metafunción influye en diversos aspectos del sistema lingüístico sin que esto signifique que se sumen paulatinamente, sino que actúan como una composición polifónica en que se entrelazan diferentes melodías semánticas.

\section{La metafunción ideacional}

El campo representa el potencial de significados del hablante como observador de la realidad y de sí mismo; expresa el contenido o el asunto a tratar; limita el concepto de la realidad. El hablante codifica su experiencia individual como miembro de una cultura.

La metafunción ideacional le permite establecer un orden a su experiencia del mundo exterior o interno, creando relaciones entre procesos, personas, circunstancias, cualidades, estados, etcétera. Los componentes básicos de esta metafunción son el proceso, el participante y las circunstancias.

La metafunción interpersonal o modal ofrece los medios para la interacción, lo que permite la expresión de posiciones sociales, de actitudes individuales, de valoraciones y la posibilidad de influir en las actitudes y en el comportamiento de otros. Este componente permite expresar las relaciones de papeles vinculados a la situación incluyendo aquellos que quedan definidos por el propio lenguaje, ya que existen lo que podríamos llamar relaciones de primer y de segundo orden. Los papeles sociales que se definen sin referencia necesaria al lenguaje pertenecen al primer orden, por ejemplo los papeles de maestro/alumno, padre/hijo, etcétera. Los papeles de segundo

\footnotetext{
${ }^{3}$ Halliday utiliza el término función para referirse al significado social de los actos verbales, pero también para referirse a componentes de significados en el sistema linguístico que determinan la organización interna del propio sistema (Halliday 1982: 98).
} 
orden son aquellos que quedan definidos por el sistema linguístico (papeles discursivos), por ejemplo los papeles de interrogador e informante, etcétera.

La metafunción textual es distinta de las otras dos metafunciones en el sentido de ser habilitadora, creadora de textos. Representa el potencial de formación de textos; es el conjunto de opciones sintácticas y de relación que permite su creación. Gracias a la metafuncióm textual, la ideacional y la interpersonal pueden realizarse.

Los tres componentes de la semántica conforman un sistema compuesto de redes y significados. De cada una de las metafunciones se derivan estructuras que se combinan y se proyectan en el nivel léxico-gramatical.

De estas metafunciones, me detendré en la textual debido a los criterios de análisis utilizados en el presente estudio.

La metafunción textual se manifiesta tanto en las cláusulas, en su estructura interna, como entre las cláusulas y el contexto de situación. Los elementos que realizan esta metafunción tienen un carácter lineal y de relación:

\section{Estructural (lineal).}

Estructura temática: tema y rema.

Estructura de información y foco: dado y nuevo.

2. Cohesión (semántica, de relación).

Referencia.

Elipsis y sustitución.

Conjunción.

Cohesión léxica.

Los componentes de las tres metafunciones se manifiestan en el sistema léxico-gramatical con fuertes restricciones internas y débiles restricciones externas. Así por ejemplo, cualquier selección hecha en el sistema de transitividad tiene un efecto importante en opciones dentro del mismo sistema pero un efecto menor en otros, tales como el modal y el temático por ejemplo.

De acuerdo al modelo Sistémico-funcional, el texto es un concepto semántico que no está compuesto de oraciones sino que se realiza por medio de oraciones. La relación entre los elementos que conforman un texto es tanto de carácter sintáctico como semántico; intra-oracional, inter-oracional y extra textual; el texto es una estructura que se realiza por medio de cláusulas, frases, grupos, etcétera, pero que es el producto de un proceso, del discurso.

A continuación explicaré brevemente las categorías de análisis que utilicé en el estudio, tomadas del modelo de Halliday y complementadas con propuestas de los lingüistas mencionados anteriormente. 


\section{La estructura temática}

Halliday utiliza el término tema siguiendo la Escuela de Praga. Lo define como el elemento en una cláusula que sirve de punto de partida y que le otorga un valor determinado como mensaje. Rema es lo que desarrolla el tema. ${ }^{4}$ Ahora bien, la posición inicial en la cláusula no explica el significado de tema, simplemente es la manera como éste se manifiesta en la lengua inglesa. Su sentido lo encontramos en cuanto a la función que cumple. El Tema es lo que el emisor selecciona como el pivote a partir del cual la cláusula se organiza como mensaje; es el elemento que indica de qué va a tratar. Así por ejemplo:

A halfpenny is the smallest English coin

The smallest English coin is a halfpenny.

Ambas cláusulas tienen el mismo significado pero difieren en cuanto a su valor como mensaje. En la primera, a halfpenny nos indica el asunto a tratar; mientras que en la segunda, el asunto es the smallest English coin. Ambas son temas en sus respectivas cláusulas.

La función del tema no se limita a la cláusula, Halliday reitera en repetidas ocasiones que si bien el tema y el rema conforman una estructura básicamente de la cláusula, existe una organización temática que se manifiesta por encima de ella. La selección del tema, lejos de ser obra del azar, tiene un valor fundamental en la organización del discurso; es el método o forma en que se desarrolla el texto (1985: 158). En este proceso, la contribución principal proviene de la estructura de las cláusulas mayores, aunque, las cláusulas menores también participan.

In the Theme-Rheme structure, it is the theme that is the prominent element...by analysing the thematic structure of a text clause by clause, we can gain an insight into its texture and understand how the writer made clear to us the nature of his underlying concerns (1985: 67).

La cláusula finita independiente en inglés puede tener una estructura temática con valor neutro (unmarked Theme) o con valor especial (marked Theme); y el tema por su parte puede ser simple o compuesto. Veámoslo en detalle.

${ }^{4}$ In English, as in many other languages, the clause is organized as a message by having a special status assigned to one part of it. One element in the clause is enunciated as the theme; this then combines with the remainder so that the two parts together constitute a message (Halliday 1985: 38). 
La cláusula finita independiente se ha definido tradicionalmente como la unidad sintáctica que contiene un sujeto y un predicado y que puede constituir una oración simple. En la cláusula declarativa, la estructura temática con valor neutro es aquella en que el sujeto (categoría interpersonal) y participante (categoría ideacional) convergen y se encuentran al inicio. En la cláusula interrogativa, el valor comunicativo determina la estructura temática. El elemento inicial, que expresa la solicitud, es el tema. El mensaje en una cláusula imperativa es "Quiero que tú hagas algo", por lo que el tema con valor neutro es el tú inicial, aunque esté omitido. Así, a manera de ejemplo:

$\begin{array}{cl}\text { TEMA NEUTRO } & \\ \text { Bears } & \text { eat honey. } \\ \text { Who } & \text { eats honey? } \\ \text { What } & \text { do bears eat? } \\ \text { (You) } & \text { Eat honey }\end{array}$

Existe, sin embargo, otra estructura temática en la lengua inglesa, muy frecuente por cierto, a la que Halliday denomina marked theme. Es una estructura especial en el sentido que rompe el esquema neutro de la lengua. El hecho de buscar patrones diferentes es indicativo del valor especial que se quiere conferir a la cláusula como mensaje. En términos generales, estaremos ante temas especiales cuando al inicio de una cláusula declarativa encontremos elementos que cumplen con funciones tales como complemento y adjunto circunstancial. Desde la perspectiva interpersonal son categorías que están, al igual que el sujeto (tema neutro), estrechamente relacionadas al finito y al predicador; y desde la perspectiva ideacional son un participante y una circunstancia relacionada con el proceso. Así, el complemento y el adjunto circunstancial pueden ser tema, con todo el peso y valor, pero con un carácter especial en cuanto a que denotan una intención particular del emisor, hecha evidente al no seguir el patrón neutro de la gramática inglesa.

El elemento más frecuente de tema especial es el adverbio, el grupo adverbial o la frase preposicional con función de adjunto circunstancial, y el elemento menos frecuente es el complemento. Esto último se debe a que el complemento se realiza típicamente por un grupo nominal, lo que equivale a una clase que de acuerdo a las posibilidades de la lengua inglesa puede funcionar como sujeto de la cláusula, pero que ha optado por la función de complemento. La posición inicial de un complemente es marcadamente forzada.

Veamos algunos ejemplos: 
TEMA NEUTRO

The duke

My aunt

TEMA ESPECIAL

That teapot,

A pudding, has given my aunt that teapot.

has been given that teapot.

the duke has given to my aunt.

the king did make.

Así como se hace la distinción entre el patrón temático con valor neutro y el patrón temático especial, es pertinente hacer la distinción entre tema simple y tema compuesto.

El primero está formado por un elemento funcional en la estructura de la cláusula, ya sea sujeto, complemento o adjunto. Estos elementos pueden, a su vez, estar constituidos por complejos unitarios, estructuras formadas por más de una estructura de la misma función y/o categoría. Los cuales son un elemento funcional de la cláusula. Por ejemplo:

\begin{tabular}{|l|l|}
\hline TEMA & REMA \\
\hline The walrus and the carpenter & were walking close at hand. \\
\hline The house by the bridge & was built by Jack \\
\hline What Jack built & was a house. \\
\hline
\end{tabular}

La estructura del tema compuesto contiene un elemento central, el núcleo (sujeto, complemento o adjunto circunstancial) más otros elementos que le anteceden y que tienen un carácter complementario. Entre ellos, encontramos las conjunciones o adjuntos conjuntivos (textual) o bien, los adjuntos modales, el verbo finito y el vocativo (interpersonal). Por adjuntos conjuntivos, Halliday se refiere a un grupo adverbial o una frase preposicional que relaciona una cláusula con otra, y aparecerán al inicio de la cláusula, cuando el emisor quiere expresar su propio juicio sobre lo que dice, o bien establecer la relación de la cláusula con lo dicho anteriormente. Veamos el siguiente ejemplo:

On the other hand, maybe

Conjuntivo

Textual

modal

interpersonal

TEMA on a week-day, it would be less crowded. núcleo

(Halliday 1985: 54). 
El tema compuesto lo podemos encontrar en cláusulas finitas independientes como en aquellas que integran una cláusula compleja. Dicha estructura muestra dos valores o funciones: su participación como organizadora del mensaje y como estructuradora del texto. En la cláusula compleja formada por una cláusula dominante y una dependiente (relación hipotáctica) puede existir más de una secuencia posible: cláusula dominante seguida por la dependiente o el orden inverso. La estructura temática neutra es la cláusula dominante seguida por la dependiente, el orden inverso indica una estructura temática especial. Así por ejemplo:

In the work of both artists, external reality is perceived as infinitely rich and varied, but (Cláusula dominante: TEMA NEUTRO)

also as capable of generating an equally rich and varied subjective reality, / which in / (Cláusula

turn devolves upon the external shaping it and giving it meaning. dependiente)

En la cláusula compleja, en donde las cláusulas que la integran tienen una relación paratáctica, la secuencia determina la función temática: la primera será tema y rema la que le siga. Así por ejemplo:

In addition, Jim is the principal figure in a richly symbolic tapestry, / so that much of (TEMA DE CLÁUSULA COMPLEJA)

what he does and says is relevant to most people at most times.

(REMA DE CLÁUSULA COMPLEJA)

En cuanto a la selección del tipo de tema en la conformación de un texto, existe una especie de gradación en la libertad temática. En una cláusula inicial, declarativa e independiente, el emisor puede seleccionar libremente la estructura. Esta libertad no es constante debido a la naturaleza de ciertas cláusulas como la interrogativa y la imperativa, o bien por las presiones ante la estructuración del texto. El concepto de tema compuesto compensa, por así decirlo, la pérdida de libertad, al extender hasta el núcleo el valor y el peso del tema (Halliday 1985: 61-62).

El análisis de la estructura temática en las cláusulas finitas permite no solamente señalar los temas sino valorarlos. Un texto no contiene, la mayoría de las veces, un solo argumento o asunto como hilo temático; el mensaje no es necesariamente monofónico. Generalmente intervienen más de uno, con diverso peso e importancia, los cuales se relacionan y se entrecruzan como una sinfonía. 
Cabe preguntarse, ¿por qué se ha utilizado la estructura temática como categoría de análisis, si la gramática funcional de Halliday maneja la estructura tema-rema como integrante de una unidad funcional? Halliday (1985) apunta a un valor temático que rebasa la cláusula, y por su parte Daneš (1974) considera al tema como un elemento crucial del enunciado en cuanto a su papel en la dinámica de la comunicación. Al rebasar la cláusula, el tema estructura a través de jerarquías y esquemas; establece una relación y, por otro lado, planifica y facilita la comprensión del contenido. "From the point of view of text organization, it is the theme that plays an important constructional role (Daneš 1974: 113)".

Brown y Yule (1983) comparten el mismo criterio de Daneš. Reconocen la doble función del tema como facilitador y planificador:

Following Daneš (1974) we shall assume that it has two main functions: i)connecting back and linking in to the previous discourse, maintaining a coherent point of view; ii) serving as a point of departure for the further development of the discourse (Brown y Yule 1983: 133).

A un nivel supraoracional, Brown y Yule proponen el principio de tematización como un marco que permite al emisor estructurar su discurso y que guía al receptor sobre el contenido del mismo.

Por su parte, el principio de estructura de la información y foco: lo dado y lo nuevo (given-new) me permitió abordar de una manera más clara la estructuración del texto al relacionar la estructura temática de la introducción con el título del ensayo. Halliday hace una clara distinción entre la estructura temática y la estructura de dado-nuevo. La unidad de información es un proceso de interacción entre lo conocido y predecible y, lo que no lo es. Lo nuevo es aquello en lo que el receptor debe fijar la atención y lo dado es aquello que el emisor considera que es conocido porque se ha mencionado anteriormente en el texto o porque es evidente en la situación a través del conocimiento compartido.

The theme is what $I$, the speaker, choose to take as my point of departure. The Given is what you, the listener, already know about or have accessible to you (Halliday 1985: 278).

De la misma manera que existe una estructura de tema-rema con valor neutro y otras con valor especial, la estructura de la información es neutra cuando el foco, el elemento nuevo, aparece al final de la cláusula. Lo que 
equivale a la coincidencia del rema con lo nuevo. Ahora bien, diversos factores pueden conjugarse para que se rompa el patrón neutro:

Within any given scenario or set of contextual conditions the speaker can exploit the potential that the situation defines, using thematic and information structures to produce an astonishing variety of rhetorical effects. He can play with the system, so to speak (Halliday 1985: 279).

Las estructuras de tema-rema y dado-nuevo son, en cierto sentido, complementarias. La estructura de información con valor neutro contiene el foco al final, y el tema se encuentra al inicio de la cláusula; el tema expresa la preocupación argumentativa del emisor y el foco el clímax o lo relevante desde el punto de vista de la información. Al combinar estas dos funciones encontramos un movimiento de disminución y crecimiento en la cláusula, disminución a partir de la prominencia del tema y crecimiento hacia el foco de la información. Halliday lo ilustra de esta manera:

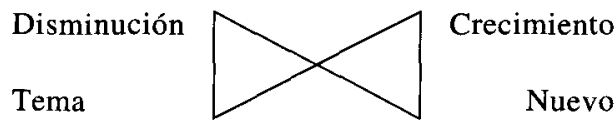

Lejos de ser un mero juego, la configuración del discurso atiende a la perspectiva funcional, al valor comunicativo que busca realizar. La organización de dado-nuevo apunta al entorno contextual; la configuración de tema-rema hacia la organización y la estructuración del texto. Ambas, por lo tanto, participan en la configuración del mensaje.

Halliday piensa que la estructura de la información (dado-nuevo) tiene una realización de carácter fonológico; sin embargo, Brown \& Yule (1983) lo estudian en cuanto a su estructura sintáctica y semántica. Su finalidad es encontrar los nexos entre la estructura sintáctica y el contexto que expliquen el valor de dicha estructura:

It is certainly the case, as Halliday has always insisted, that information status is determined, not by the structure of discourse but by the speaker. It is also certainly the case that there are no "rules" for the specification of "new" or "given" status by the speaker. There are, however, regularities (Brown \& Yule 1983: 9).

Proponen que la información se puede considerar como nueva, inferible o evocada por medio del uso de ciertas categorías sintáctico-pragmáticas que el emisor utiliza de acuerdo al tipo de información que transmite. 
$A$ (+ propiedades) para información nueva.

The (+ propiedades) para información inferible.

It (+ propiedades) para información inferible.

Pronombres exofóricos que remiten al contexto físico de la situación para información evocada situacional.

Pronombres, elipsis o bien una referencia mínina, para información evocada textual y desplazada.

Los criterios de Brown \& Yule (1983) me permitieron incursionar en el estudio de esta estructura funcional para establecer los nexos entre el título del ensayo y la estructura o secuencia temática de la introducción.

Sobre la base de estos criterios me propuse describir la estructura temática de las cláusulas en la introducción y la relación semántica entre los temas sin considerar la relación con los remas. De la relación entre los temas obtuve una configuración que junto con el título, considero, informan sobre el método o la manera en que el texto se desarrolla en cuanto a su contenido. La relación entre los temas a lo largo de la introducción del ensayo la establecí por medio de los criterios de cohesión de Halliday y Hasan (1976). ${ }^{5}$

El título juega también un papel muy importante como elemento de tematización, ya que ofrece al escritor un punto de partida para estructurar su texto y al lector, expectativas sobre el contenido. Al respecto Brown y Yule señalan:

This expectation-creating aspect of thematization, especially in the form of a title, means that thematised elements provide not only a starting point around which what follows in the discourse is structured, but also a starting point which constrains our interpretation of what follows (1983: 139).

El título cumple con la función de guía en el desarrollo subsecuente del texto y, considero yo, establece dicha relación por medio de la configuración temática. Ambos juegan un papel muy importante como organizadores del texto.

${ }^{5}$ A través del concepto de cohesión, Halliday y Hasan (1976) explican los vínculos de carácter semántico entre dos o más elementos en el texto. La cohesión es parte del sistema de la lengua, de la metafunción textual. Los recursos linguísticos de que se vale son repeticiones, omisiones, uso de ciertas palabras o estructuras. Dependiendo del tipo de relación que estos recursos establecen con otros elementos en el texto, la cohesión puede ser anafórica o catafórica. Los medios por lo que se realiza son la referencia, la elipsis, la sustitución, la conjunción y la organización léxica. 
Para establecer su relación es necesario partir de la descripción del título. La estructura formal de los títulos en el tipo de texto que concierne este trabajo es la de un grupo nominal: ${ }^{6}$

A noun phrase may be an indeterminately long structure having a noun as head, preceded by other words such as an article, an adjective, or another noun, and followed by a prepositional phrase or by a relative clause (Quirk, Greenbaum, Leech \& Svartvik, 19972: 414).

El grupo nominal tiene una gran capacidad de información, de aquí la gran diversidad de combinación de elementos estructurales.

La metafunción semántica relativa a la organización de la experiencia, la ideacional, está claramente presente en el grupo nominal. Halliday (1985) define al grupo nominal como la expresión de una experiencia que especifica una clase de "cosa" (thing) en cuanto a categoría, inclusión y relación dentro de la clase, por medio de deícticos, numerales, epítetos y clasificadores. La especificación no se presenta únicamente antecediendo a la cosa (premodificación); también existe la modificación posterior, una frase o una cláusula que califican. Por ejemplo:

\begin{tabular}{|l|l|l|l|l|l|}
\hline DEITICO & NUMERAL & ESPITETO(s) & CLASIFICADOR & “COSA" & ClASIFIC. \\
\hline Those & two & splendid, old & electric & TRAINS & $\begin{array}{l}\text { with } \\
\text { pantographs }\end{array}$ \\
\hline
\end{tabular}

En cuanto a su función, Halliday (1985) considera a los títulos, junto con los nombres de productos, avisos y algunos tipos de instructivos como pequeños textos, que cumplen con una función particular y tienen una dinámica propia.

Parte del valor de los títulos lo tenemos que inferir a través de la implicación, de los nexos que existen entre el "pequeño texto" y el contexto de

${ }^{6}$ TÍTULOS DEL CORPUS

1. "The Perception of Reality as an Act of 'Reading' (An examination of one aspect in the art of Proust and Joyce)".

2. "The Character of Jim in Conrad's Lord Jim".

3. "The story of the Epic of Gilgamesh".

4. "The struggle to Surface in the Water of Sonny's Blues".

5. "A nightmare of Nightmares. A fusion of Literary Awareness and Political Consciousness in Julio Cortázar”. 
situación en que aparecen. Las implicaciones "implicatures" (Grice 1975) se derivan parcialmente del significado literal complementado por el contexto, que ofrece el valor que el emisor/receptor confieren al enunciado. Por su parte, Widdowson (1983) propone la existencia de marcos de referencia (schemata) que permiten establecer expectativas y posibles interpretaciones del contenido con que nos enfrentamos, ya que representan nuestra experiencia, el conocimiento adquirido. Así por ejemplo, en el título:

"The Character of Jim in Conrad's Lord Jim"

el lector, al considerarlo el título de un ensayo sobre literatura, presupone que Conrad es el autor de la obra objeto del estudio.

Por otra parte, es frecuente encontrar en los títulos un predicado "latente", implícito y recuperable, presupuesto en el sentido de Strawson (1971). ${ }^{7}$ Esto se debe a su compleja función semántica y apunta dentro de un análisis más fino a proponer una estructura de tema y rema en el título.

Los títulos con frases preposicionales muestran claramente este fenómeno. Cabe mencionar que para la gramática de Halliday (1985), la preposición se asemeja a un verbo menor y es factible en algunos casos expresar su significado valiéndose de un verbo no-finito. Así, a manera de ejemplo, tenemos el siguiente título del corpus estudiado:

"The Perception of Reality as an Act of 'Reading"

Título que se puede reformular como:

"The Perception of Reality Intended as an Act of 'Reading",

En el estudio realizado, el título se describe como un grupo nominal compuesto por una clase de cosa o cabeza (head) que se especifica con premodificadores y/o posmodificadores. Asimismo, es considerado como un pequeño texto con una dinámica propia, cuyo valor lo tenemos que inferir, en algunos casos, a través de la implicación y de un predicado "latente", debido a la compleja función semántica que cumple.

Aplicación. El corpus para el análisis lo conforman ensayos de carácter académico, que aparecen en publicaciones de estudios sobre literatura comparada, en el Anuario de Letras Modernas de la UNAM y en libros de texto para la enseñanza de redacción en inglés.

${ }^{7}$ Es precisamente en este punto por lo que podemos distinguir el título de los grupos nominales convencionales. Como lo muestra Strawson, el punto principal de una presuposición (desde el punto de vista comunicativo) es que permite al hablante, aun en los casos que no sea cierto, referirse al argumento de la proposición que expresa. Es decir, la presuposición tiene un valor claramente distinto a la proposición expresada. En un título la presuposición tiene un valor primordial, es como si lo que se presupone se afirmara. 
Éstos son:

Pimentel, L. A., "The Perception of Reality as an Act of 'Reading"' (An examination of one aspect in the art of Proust and Joyce", en Comparative Literature Studies. [s. e. s. 1].

Broad, Ch., "A Nightmare of Nighmares. A fusion of Literary Awareness and Political Consciousness in Julio Cortázar", en Anuario de Letras Modernas, vol. 2. México, UNAM, Facultad de Filosofía y Letras, 1984.

Roberts, E., “The Character of Jim in Conrad's Lord Jim", en Writing Themes about Literatures, Englewood Cliffs, N. J., Prentice-Hall, 1964.

Roberts, E., "The Story of the Epic of Gilgamesh", en Writing Themes About Literatures. Englewood Cliffs, N. J., Prentice-Hall, 1964.

Geoffrey, C., "The Struggle to Surface in the Water of Sonny's Blues", en The Norton Introduction to Literature. C. Bain, J. Beaty y P. Hunter, eds. Nueva York, Norton \& Co., 1982.

De estos ensayos me permito presentar el análisis del primero y segundo.

Las categorías de análisis aplicadas son las siguientes:

\section{La estructura temática en las cláusulas finitas.}

Se señalarán los diversos tipos de estructuras: neutra, especial, simple y compuesta.

2. La configuración temática de la introducción.

Se considerará la estructura temática con un valor que trasciende a la cláusula finita al participar en la configuración semántica de la introducción.

3. El título y la relación con la configuración temática.

Se describirá el título y se establecerá la relación con la configuración temática de la introducción.

\section{La estructura de la información.}

Se describirá el valor de las estructuras temáticas en cuanto a su relación con la estructura de la información.

Las convenciones utilizadas en el análisis de la estructura temática de la introducción de los ensayos son las siguientes:

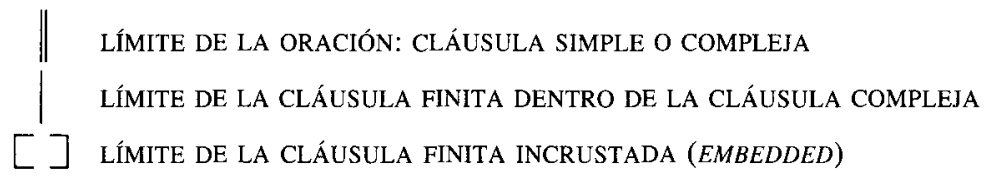




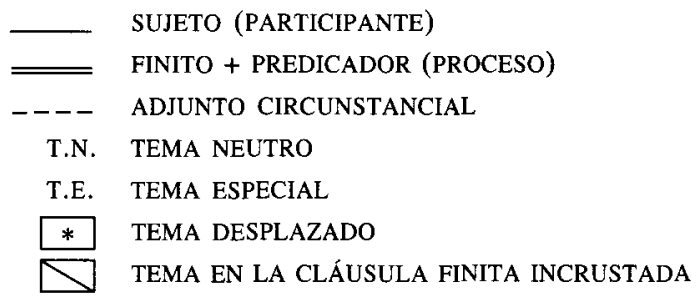

\section{Análisis de la muestra 1}

"The Perception of Reality as an Act of 'Reading' (An examination of one aspect in the art of Proust and Joyce)"

In Proust and Joyce, literature turns inwards to explore the dark regions of the mind. The landscapes of consciousness are painted and their relationship to the world outside carefully traced. In the work of both artists, external reality is perceived as infinitely rich and varied, but also as capable of generating an equally rich and varied subjective reality, which in turn devolves upon the external world shaping it and giving it meaning. For both artists, the world without can be "read" for meaning, for a meaning which is ultimately an articulate image of the self.

\section{Análisis de la estructura temática en la introducción de la muestra 1}

1. In Proust and $\underline{\text { Joyce, }}$ literature turns inwards to explore the dark regions of the mind.

CLÁUSULA INDEPENDIENTE

TEMA ESPECIAL

\begin{tabular}{|l|l|l|l|l|}
\hline 2. The landscapes of consciousness are painted & \multicolumn{2}{l|}{ and their relationship to the world outside } \\
\hline \multicolumn{2}{|l|}{ CLÁUSULA PRIMARIA } & CLÁUSULA SECUNDARIA \\
\hline \multicolumn{2}{|l|}{ TEMA NEUTRO EN LA C. COMPLEJA } & & \multicolumn{3}{|l|}{ NÚCLEO T. COMPUESTO } & \\
\hline & TEMA NEUTRO & &
\end{tabular}




\begin{tabular}{|c|c|c|c|}
\hline carefully traced. & \multicolumn{3}{|c|}{ 3. In the work of both artists, external reality is perceived as infinitely } \\
\hline & \multicolumn{3}{|c|}{ CLÁUSULA DOMINANTE } \\
\hline & \multicolumn{3}{|c|}{ TEMA EN O. COMPLEJA } \\
\hline & TEMA ESPECLAL & * & \\
\hline
\end{tabular}

\section{rich and varied, but also as capable of generating an equally rich and varied subjective}

\begin{tabular}{|l|l|||l|}
\hline reality, & which in turn devolves upon the external world shaping it and giving it meaning. & \\
\hline & CLÁUSULA SUBORDINADA & \\
\hline & & \\
\hline & TEMA NEUTRO & \\
\hline
\end{tabular}

4. For both artitsts, the world without can be "read" for meaning, for a meaning CLÁUSULA INDEPENDIENTE (con...

TEMA ESP.

$*$

$\underline{\text { which }} \underline{\underline{=}}$ ultimately an articulate image of the self.

CLÁUSLIA INCRUSTADA 


\section{La configuración temática}

\begin{tabular}{|c|c|c|c|c|c|c|c|}
\hline \multirow{2}{*}{ Sentence } & \multicolumn{3}{|c|}{ TEMA NEUTRO } & \multicolumn{3}{|c|}{ TEMA ESPECIAL } & \multirow{2}{*}{$\begin{array}{c}\text { Tipo y función } \\
\text { de las } \\
\text { cláusulas }\end{array}$} \\
\hline & Simple & $\begin{array}{l}\text { Cor } \\
\text { Antece. }\end{array}$ & $\begin{array}{l}\text { Resto } \\
\text { núcleo }\end{array}$ & Simple & Compuesto & $\begin{array}{c}\text { Tema } \\
\text { desplazado }\end{array}$ & \\
\hline 1 & & & & $\begin{array}{l}\text { In Proust } \\
\text { and Joyce }\end{array}$ & & Literature & $\begin{array}{c}\text { Cláusula } \\
\text { independiente }\end{array}$ \\
\hline \multirow{2}{*}{2} & $\begin{array}{c}\text { The } \\
\text { landscapes } \\
\text { of } \\
\text { conscious- } \\
\text { ness }\end{array}$ & & & & & & $\begin{array}{c}\text { Cláusula } \\
\text { primaria } \\
\text { (tema) }\end{array}$ \\
\hline & & And & $\begin{array}{l}\text { Their } \\
\text { relation- } \\
\text { ship to the } \\
\text { world } \\
\text { outside }\end{array}$ & & & & $\begin{array}{l}\text { Cláusula } \\
\text { secundaria } \\
\text { (rema) }\end{array}$ \\
\hline \multirow{2}{*}{3} & & & & $\begin{array}{c}\text { In the } \\
\text { work of } \\
\text { both artists }\end{array}$ & & $\begin{array}{c}\text { External } \\
\text { reality }\end{array}$ & $\begin{array}{c}\text { Cláusula } \\
\text { dominante } \\
\text { (tema) }\end{array}$ \\
\hline & $\begin{array}{l}\text { Which } \\
\text { (subjective } \\
\text { reality) }\end{array}$ & & & & & & $\begin{array}{l}\text { Cláusula } \\
\text { dependiente } \\
\text { (rema) }\end{array}$ \\
\hline \multirow{2}{*}{4} & & & & $\begin{array}{l}\text { For both } \\
\text { artists }\end{array}$ & & $\begin{array}{l}\text { The word } \\
\text { without }\end{array}$ & $\begin{array}{l}\text { Cláusula } \\
\text { principal }\end{array}$ \\
\hline & $\begin{array}{l}\text { Which } \\
\text { (a } \\
\text { meaning) }\end{array}$ & & & & & & $\begin{array}{c}\text { Cláusula } \\
\text { incrustada }\end{array}$ \\
\hline
\end{tabular}

Por medio del análisis de la estructura temática de las cláusulas reconocemos temas especiales en tres de las cuatro oraciones que integran la introducción. Éstos son temas relativos a la OBRA DE PROUST Y JOYCE. Es interesante notar que aun en la oración inicial, declarativa e independiente, se encuentra un tema especial, el cual se repite en la cláusula dominante de la tercera oración y en la cláusula principal de la cuarta oración. La autora selecciona libremente esta estructura, señalando una intencionalidad al otorgar un valor argumentativo en el desarrollo de la introducción a LA OBRA DE PROUST Y JOYCE, como el asunto más importante a tratar.

Existe una relación evidente entre los temas especiales, que se logra a través de la cohesión léxica. En la cláusula dominante de la oración tres, el tema especial contiene como núcleo de la frase circunstancial THE WORK, como preocupación argumentativa Así, propongo LA OBRA DE PROUST Y JOYCE como tema A. 
Encuentro como temas neutros a LA DESCRIPCIÓN DE LO CONSCIENTE Y SU RELACIÓN CON EL MUNDO EXTERIOR en la oración dos (una cláusula compleja en relación paratáctica). En la oración tres, la cláusula dependiente tiene WHICH como tema, el cual nos remite a la REALIDAD SUBJETIVA. Por lo tanto, LO CONSCIENTE Y LO SUBJETIVO Y, SU RELACIÓN CON EL MUNDO EXTERNO, se considera como tema $B$.

Así, propongo que la forma en que esta introducción se desarrolla es delimitando en la obra de Proust y Joyce el asunto a tratar: LO CONSCIENTE Y LO SUBJETIVO Y SU RELACIÓN CON EL MUNDO EXTERNO.

En cuanto al título "THE PERCEPTION OF REALITY AS AN ACT OF 'READING", tiene la estructura formal de un grupo nominal, que podemos describir de la siguiente manera:

\begin{tabular}{|l|l|l|}
\hline THE & PERCEPTION & OF REALITY AS AN ACT OF "READING" \\
\hline Pre-modificador & Cosa-cabeza & Posmodificadores \\
\hline
\end{tabular}

Si consideramos el título como un "pequeño texto" que trasciende el valor de un mero grupo nominal, lo podemos analizar como un argumento con su predicado: THE PERCEPTION OF REALITY (INTENDED) AS AN ACT OF "READING".

Por su parte, el subtítulo (AN EXAMINATION OF ONE ASPECT IN THE ART OF PROUST AND JOYCE) es también un grupo nominal, AN EXAMINATION es una implicación convencional, el quehacer que la autora, como estudiosa de la literatura, se propone realizar.

El título y el subtítulo se relacionan semánticamente: ONE ASPECT establece cohesión léxica con THE PERCEPTION OF REALITY AS AN ACT OF "READING". El empleo de artículos indefinidos parece sugerir que el subtítulo debiera anteceder al título.

En cuanto a la relación del título con la configuración temática de la introducción, encuentro que el tema neutro se relaciona con parte del núcleo del título: OF REALITY que se refiere a la DESCRIPCIÓN DE LO CONSCIENTE Y SU RELACIÓN CON EL MUNDO EXTERNO.

El tema especial: PROUST Y JOYCE / SU OBRA se relaciona con las dos últimas frases del subtítulo: IN THE ART OF PROUST AND JOYCE.

Encuentro que la relación entre el título y la configuración temática de la introducción no incluyen todos los componentes del título. Tal es el caso de PERCEPTION, EXAMINATION Y ACT OF “READING". Podemos, sin embargo, 
considerar EXAMINATION Y ACT OF "READING" como implicaciones convencionales: el quehacer del creador literario y del estudioso del texto literario. Para concluir, el tema neutro $\mathrm{B}$ se relaciona con el núcleo argumentativo del título y, el tema especial A se relaciona con el subtítulo, en cuanto a la circunstancia que restringe y complementa al núcleo: EXAMINATION.

La estructura de la información. La relación entre el título y la progresión temática de la introducción nos hace pensar en la convergencia de los temas con lo dado, una estructura de la información con valor neutro (Halliday 1985).

De acuerdo a la taxonomía de Brown y Yule (1983), el artículo definido, el adjetivo posesivo y la elipsis en el título señalan información dada (evocada e inferible); sin embargo, es interesante notar que la primera oración de la introducción tiene como tema especial a IN PROUST AND JOYCE, repitiendo los mismos apelativos del título y sugiriendo una independencia de la introducción con respecto al título El apego al patrón neutro en la estructura de la información, lo podemos justificar en cuanto al carácter didáctico del ensayo, que confiere una función "facilitadora" al título y a la introducción.

\section{Análisis de la muestra 2}

\section{"A Nightmare of Nightmares. A fusion of Literary Awareness and Political Consciousness in Julio Cortázar"}

Julio Cortázar expresses his concern in his introduction to El libro de Manuel for the problems of reconciling a political awareness with a literary consciousness. He explains how he later discovered that "el sueño era también parte del libro y contenía la clave de esa convergencia de actividades hasta entonces disimiles". In a novel patched with newspaper cutting and dealing explicitly with political topics among others, it is precisely the dream, a subversive pattern running throughout the work, that unites the author's literary and political ambition. A nightmare, meanwhile, provides the central theme and key to this convergence of activities in "Pesadillas", a short story in his recent collection Deshoras. The apparent nightmare of a young girl, Mecha, in coma, affects all those surrounding her within the house and transcends this environment to fuse with the exterior political reality. In this case, the political situation is treated evasively as the clinical nightmare captures narrative attention. However, the indirectness in approach to this onírico-political phenomenon reveals its importance and allows Cortázar to bridge the gap between political and literary awareness. 


\section{Análisis de la estructura temática en la introducción de la muestra 2}

\section{Juljo Córtazar expresses his concern in the introduction to El libro de Manuel for the problem of}

\begin{tabular}{|l|l|}
\hline \multicolumn{2}{|c|}{ CLÁUSULA INDEPENDIENTE } \\
\hline TEMA N. & \\
\hline
\end{tabular}

\begin{tabular}{|l||l|l|}
\hline reconciling a political awareness with a literary consciousness. & 2. He explains & how he later \\
\hline & CL. IND. & CLÁUSULA IND. \\
\hline & T.N. & \\
\hline
\end{tabular}

\begin{tabular}{|l|l|}
\hline $\begin{array}{l}\text { discovers } \\
\text { that "el sueño era también parte del libro y contenía la clave de esa convergencia de }\end{array}$ \\
\hline CRUST. & CLÁUSULA INCRUSTADA \\
\hline & \\
\hline
\end{tabular}

\begin{tabular}{|l||l|}
\hline actividades hasta entonces disímiles" & 3. In the novel patched with newspaper cuttings and dẹling \\
\hline & CL. MNEPENDIENTE \\
\hline & T. ESPECLAL \\
\hline
\end{tabular}

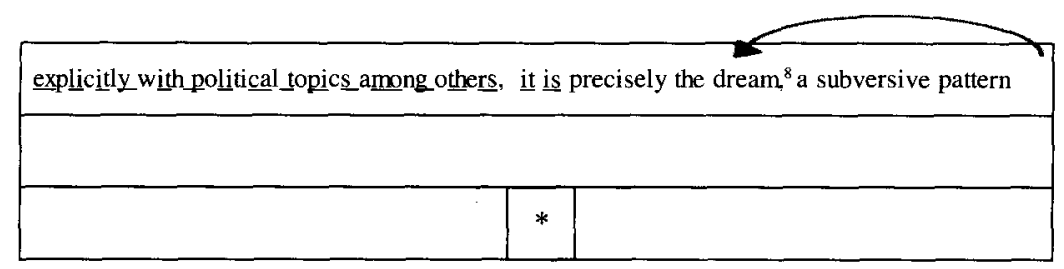

${ }^{8}$ It es el sujeto gramatical, posible tema que ha sido desplazado por una frase circunstancial que ocupa en la oración la posición del tema. Sin embargo, es interesante resaltar que it is precisely the dream contiene un tema predicado por medio de lo que 


\begin{tabular}{|c|c|c|}
\hline running thoughout the work, & that unites the author's literary and political ambition. & 4. A nightmare, \\
\hline & CL. INCRUSTADA & CLÁUSULA \\
\hline & & T. NEUTRO \\
\hline
\end{tabular}

meanwhile, provides the central theme and key to this convergence of activities in "Pesadillas", INDEPENDIENTE

\begin{tabular}{|l|l|l|}
\hline a short story in his recent collection Deshoras. & 5. The apparent nightmare of a young girl, Mecha, \\
\hline & CL. PRIMARIA: TEMA EN CL. COMPLEJA \\
\hline & TEMA NEUTRO & \\
\hline
\end{tabular}

\begin{tabular}{|l|l|l|l|}
\hline \multicolumn{1}{|l|}{ in coma $\underline{\underline{\text { affects }}}$ all those sorrounding her within the house } & and (it) transcends this evironment to \\
\hline & CL. SECUNDARIA: \\
\hline & & TEMA MÚLTIPLE \\
\hline & Conj. T.N. & \\
\hline
\end{tabular}

\begin{tabular}{|l|l|l|l|l|}
\hline fuse with the exterior political reality. & \multicolumn{2}{|l|}{ 6. In this çase, the political situation is treated evasively } & as \\
\hline & \multicolumn{2}{|c|}{ CLÁUSULA NNDEPENDIENTE } & \\
\hline & T. ESPECIAL & $*$ & & \\
\hline
\end{tabular}

Halliday llama cleft sentence; de tal manera que the dream, tema predicado de la cláusula, tiene un marcado peso informativo y hasta cierto punto un valor temático. Cabe mencionar que El sueño aparece como tema en una cláusula incrustada de la oración 2. 


\begin{tabular}{|l|l|l|l|}
\hline \multicolumn{2}{|l|}{ the clinical nightmare captures narrative attention. } & \multicolumn{2}{l|}{ However, the indirectness in approach to this } \\
\hline & CL. PRIMARIA: TEMA NEUTRO EN CL. \\
\hline & TEMA MÚLTIPLE \\
\hline & Conj. Adj. & Tema tópico & \\
\hline
\end{tabular}

\begin{tabular}{|c|c|}
\hline oneirico-political phenomenon reveals its importance & and (it) allows Cortázar to bridge the gap \\
\hline \multirow[t]{2}{*}{ COMPLEJA } & CLÁUSULA SECUNDARIA \\
\hline & TEMA MÚLTIPLE \\
\hline & Conj.T.N. \\
\hline
\end{tabular}

\begin{tabular}{|l||l|}
\hline between political and literary awareness. & \\
\hline & \\
\hline & \\
\hline
\end{tabular}

${ }^{9}$ However cumple una función compleja en el sentido de conectar con lo dicho anteriormente y tiene un valor adverbial en cuanto que expresa el sentir sobre lo que se dice. Halliday la llama conjunctive adjunct adversativo Dicha categoría no permite que sea considerada como tema especial. 
La configuración temática en la muestra 2

\begin{tabular}{|c|c|c|c|c|c|c|c|c|}
\hline \multirow{3}{*}{ Oración } & \multicolumn{3}{|c|}{ Tema neutro } & \multicolumn{4}{|c|}{ Tema especial } & \multirow{2}{*}{$\begin{array}{l}\text { Tipo y } \\
\text { función de } \\
\text { las cháusulas }\end{array}$} \\
\hline & \multirow[t]{2}{*}{ Simple } & \multicolumn{2}{|c|}{ Compuesto } & \multirow[t]{2}{*}{ Simple } & \multicolumn{2}{|c|}{ Compuesto } & \multirow{2}{*}{$\begin{array}{l}\text { Tema } \\
\text { despla- } \\
\text { zado }\end{array}$} & \\
\hline & & Antecedentes & Núcleo & & Antecedentes & Núcleo & & \\
\hline 1 & J. & & & & & & & $\begin{array}{l}\text { Cláusula } \\
\text { independiente }\end{array}$ \\
\hline 2 & $\begin{array}{l}\text { He (Cor- } \\
\text { tázar) }\end{array}$ & & & & & & & $\begin{array}{l}\text { Cláusula } \\
\text { independiente }\end{array}$ \\
\hline 3 & & & & $\begin{array}{l}\text { IN A NOVEL } \\
\text { PATCHED WTTH } \\
\text { CUTTINGS AND } \\
\text { DEALING } \\
\text { EXPUCTLL Y } \\
\text { WITH } \\
\text { POLIICAL ... } \\
\text { OTHERS }\end{array}$ & & & $\begin{array}{l}\text { It } \\
\text { (dream) }\end{array}$ & $\begin{array}{l}\text { Cláusula } \\
\text { independiente }\end{array}$ \\
\hline 4 & $\begin{array}{l}\text { A NMGHT } \\
\text { MARE }\end{array}$ & & & & & & & $\begin{array}{l}\text { Cláusula } \\
\text { independiente }\end{array}$ \\
\hline \multirow[t]{2}{*}{5} & $\begin{array}{l}\text { THE } \\
\text { APPARENT } \\
\text { NGGT } \\
\text { MARE OF } \\
\text {...N } \\
\text { COMA }\end{array}$ & & & & & & & $\begin{array}{l}\text { Cláusula } \\
\text { primaria }\end{array}$ \\
\hline & & and & $\begin{array}{l}\text { T (apparent } \\
\text { nightmare) }\end{array}$ & & & & & $\begin{array}{l}\text { Cláusula } \\
\text { secundaria }\end{array}$ \\
\hline 6 & & & & $\begin{array}{l}\text { In this case } \\
\text { (Pesadillas) }\end{array}$ & & & $\begin{array}{l}\text { The } \\
\text { political } \\
\text { situation }\end{array}$ & $\begin{array}{l}\text { Cláusula } \\
\text { independiente }\end{array}$ \\
\hline \multirow{2}{*}{7.} & & however & $\begin{array}{l}\text { the } \\
\text { indirenctness } \\
\text { In approach } \\
\text { to }\end{array}$ & & & & & $\begin{array}{l}\text { Cláusula } \\
\text { primaria }\end{array}$ \\
\hline & & and & $\begin{array}{l}\text { It (the } \\
\text { indirectness } \\
\text { in aproach } \\
\text { to) }\end{array}$ & & & & & $\begin{array}{l}\text { Cláusula } \\
\text { secundaria }\end{array}$ \\
\hline
\end{tabular}

El análisis de las estructuras temáticas de las cláusulas finitas nos muestra como tema neutro a JULIO CORTÁZAR, la que llamaremos tema A. UNA PESADILLA Y LA PESADILLA APARENTE DE UNA JOVEN EN ESTADO DE COMA son también temas neutros en dos cláusulas y en el núcleo de un tema compuesto en la cláusula secundaria de la oración 5: tema B. UNA NOVELA Y EL CUENTO "PESADILLAS" son temas especiales en dos cláusulas independientes: tema C. LO INDIRECTO DEL ACERCAMIENTO AL FENÓMENO ONÍRICOPOLítico es núcleo en dos temas compuestos en la oración 7: tema D. 
El método de desarrollo es complejo e interesante. JULIO CORTÁZAR, LA PESADILLA EN ALGUNAS OBRAS COMO UN ACERCAMIENTO INDIRECTO AL FENÓMENO ONÍRICO-POLÍTICO.

Es interesante detenerse en la última oración de esta muestra en la que se indica, con un tono enfático (con el uso de however), el valor del acercamiento al fenómeno onírico-político. Tal pareciera que la autora del ensayo reserva para el final de la introducción el valor argumentativo de dicho acercamiento.

En cuanto al título está compuesto de dos grupos nominales. El segundo aclara y explica al primero.

\begin{tabular}{|l|l|}
\hline A nightmare of nightmares. & $\begin{array}{l}\text { A fusion of literary awareness and political } \\
\text { consciousness in Julio Cortázar. }\end{array}$ \\
\hline COSA POSMODIFICACIÓN & COSA POSMODIFICACIÓN \\
\hline
\end{tabular}

La relación entre el título y la configuración temática es la siguiente: el núcleo del primer grupo nominal del título se relaciona con el tema B (UNA PESADILLA Y LA PESADILLA APARENTE DE UNA JOVEN EN ESTADO DE COMA). El núcleo del segundo grupo nominal: A FUSION OF LITERARY AWARENESS AND POLITICAL CONSCIOUSNESS se relaciona con el tema D (LO INDIRECTO DEL ACERCAMIENTO AL FENÓMENO ONÍRICO-LITERARIO). La última frase del título IN JULIO CORTÁZAR se relaciona con los temas A y C (JULIO CORTÁZAR EN EL LIBRO DE MANUEL Y DESHORAS).

En cuanto a la estructura de la información encontramos un artículo indefinido en un tema neutro (A NIGHTMARE), así como la expresión de propiedades en un tema neutro (THE APPARENT NIGHTMARE OF A YOUNG GIRL IN COMA) y en uno especial (IN A NOVEL PATCHED WITH CUTTINGS AND DEALING EXPLICITLY WITH POLITICAL TOPICS AMONG OTHERS), elementos que señalan información nueva o bien fuera de uso. Por otro lado, encontramos un adjetivo demostrativo (THIS), pronombres (HE, IT) y un artículo definido (THE), los cuales indican información dada: inferible o evocada textualmente. Así, en cuanto a la estructura de la información, los temas en la introducción no siempre convergen con lo dado, aún cuando la información esté presente en el título. Tal es el caso de los temas B y C.

Beaugrande \& Dressler (1986) proponen tres principios regulativos que regulan la comunicación textual: eficiencia (efficiency), eficacia 
(effectiveness) y apropiado (appropriateness). Considero que esta muestra es un interesante juego entre eficiencia y eficacia, dándole posiblemente más peso a la última. La eficiencia contribuye a facilitar la comprensión del texto, cuando las operaciones se llevan a cabo con una leve carga para su comprensión; la eficacia promueve la comprensión a profundidad del texto. Estos dos principios parecieran actuar uno en contra del otro: sencillez, claridad $v s$. complejidad, profundidad. Sin embargo, un balance adecuado sería aquel que evite el aburrimiento ante lo demasiada obvio y la incomprensión ante lo demasiada complejo Un juego entre los dos principios resultará en textos atractivos y comunicativos, como el texto que nos concierne.

\section{Conclusiones generales}

Como resultado del análisis realizado en las cinco muestras que conformaron el corpus (incluyendo los dos análisis presentados aquí), encontré que los temas se relacionan entre sí, por medio de vínculos sintáctico-semánticos, lo que me permite proponer la existencia de una configuración temática de las cláusulas finitas (de dos a cuatro temas por introducción). Dicha configuración nos muestra que la selección del contenido en la parte inicial de las cláusulas no es caótica e inconexa, sino por el contrario, es una evidencia que la organización responde a una preocupación argumentativa. En dos de las muestras (las aquí presentadas), el método de desarrollo es delimitando el ámbito del análisis en el espacio y el tiempo y, señalando el asunto a tratar. En las otras tres muestras, se propone el asunto y se informa en qué sentido o aspecto será considerado.

A partir del análisis del título busqué el vínculo con la estructura temática. Si bien es cierto que no es posible señalar una relación explícita y formal entre todos los elementos que integran el título y los temas de las cláusulas finitas de la introducción, sí encontré una conexión (por cohesión) en la mayoría de las muestras analizadas. Los temas especiales en tres de las muestras se relacionan con frases posmodificadoras en el título y se refieren a las circunstancias o a las cualidades que restringen y complementan el núcleo del título. En cuatro de las muestras, los temas neutros de las cláusulas finitas de la introducción se relacionan con el núcleo del título. Sin embargo, encontré temas neutros que se relacionan con frases modificadoras del título (en dos muestras), y temas especiales que lo hacen con el núcleo del título (en dos muestras). Asimismo, encontré temas que no tienen referente en los títulos (en dos muestras) y componentes de los títulos que no se retoman en la estructura temática de la introducción (una muestra). Esto me 
lleva a considerar que no es posible establecer una dependencia entre el título y la introducción y que ambos tienen un valor propio. Cabe mencionar que en tres de las muestras encontré en la introducción apelativos (PROUST Y JOYCE, GILGAMESH y SONNY'S BLUES) contenidos en los títulos, en lugar de un referente personal, que podríamos haber esperado si el título y la introducción estuvieran más ligados. No hay que olvidar que Halliday considera al título como un "pequeño texto".

Encontré necesario recurrir a las "implicaciones" tanto para establecer la estructura temática como para hallar los nexos entre el contenido del título y los temas de la introducción.

En cuanto a la estructura de la información, me permitió apoyar la tesis de la existencia de una estructuración del ensayo para facilitar su comprensión. Solamente en la muestra 2 encontré en la introducción temas que expresan información nueva o fuera de uso. Pienso que el principio de claridad (eficiencia de acuerdo a Beaugrande \& Dressler) no es el único que rige la estructuración de las introducciones en ensayos de crítica literaria.

Estoy muy lejos de pretender que el estudio realizado sea de carácter exhaustivo; éste es sólo el inicio de una análisis desde una perspectiva funcional. Cabe reconocer que junto con los resultados obtenidos, me han surgido dudas con relación al modelo Sistémico-funcional, a la gramática de Halliday y a su aplicabilidad. Así por ejemplo, partí de categorías claras y distintivas, con una realización precisa en el nivel lexicogramatical, sin embargo, al aplicarlas, parte de la claridad se esfumó. Halliday da todo el peso de la organización argumentativa al tema y describe sus posibles realizaciones gramaticales; considera al tema compuesto como una categoría tan precisa como las demás. Sin embargo, al incorporar todos los elementos que anteceden al núcleo del tema compuesto, se pierde su carácter distintivo. ¿Es esto el resultado de una comprensión y/o aplicación defectuosa del modelo? O bien: ¿es la misma naturaleza de la lengua que no permite gran especificidad y un modelo que abarca desde la semiótica social hasta la gramática no puede lograr criterios tan precisos? Habrá, indudablemente, que proseguir el estudio y considerar que el propio Halliday está consciente de la naturaleza del discurso y así dice:

Discourse is a multi-dimensional process $[\ldots]$ as the realization of semiotic orders "above" the language, may contain in itself all the inconsistencias, contradictions and conflict that can exist within and between such higher order semiotic system (1985: 318).

Dos fueron las preocupaciones fundamentales al elaborar este trabajo: incursionar en el análisis del texto desde una perspectiva funcional e iniciar 
una descripción del ensayo de crítica literaria que apoye a los estudiantes de Letras Inglesas en la valoración y redacción del mismo. A pesar de las dudas surgidas, pienso que una perspectiva funcional, aunque compleja, es una posible respuesta para este tipo de tarea, ya que el funcionalismo considera al texto como una unidad sintáctica y semántica, vinculada al contexto extralingüístico; lo explica en cuanto a su cometido comunicativo y a su naturaleza social y expresiva. El funcionalismo rebasa los criterios tradicionales del análisis lingüístico y sienta las bases para una descripción integral del lenguaje. En cuanto a una posible aplicación pedagógica, creo que se podría pensar en proveer al estudiante de Letras Inglesas, a partir del modelo utilizado, con una gramática de "identificación", tal y como la define Rall (1980): una gramática de reconocimiento de las variaciones sintácticas en cuanto a su valor semántico y discursivo. En este caso, una gramática de identificación de la manera en que la introducción y el título están organizados para satisfacer las exigencias del ensayo de crítica literaria. Por otra parte, la aplicación es el único medio para informarse sobre los aciertos de las categorías empleadas. Este cometido se lograría al ofrecer al estudiante una explicación del marco teórico que fundamenta el criterio de análisis y una descripción de las categorías. A partir de este conocimiento, y a través de un contacto con textos auténticos, se buscaría desarrollar una sensibilidad, una manera de explorar la organización discursiva para valorarla y así poder utilizarla en sus propios ensayos.

Como espero haberlo mostrado en este trabajo, el estudio de la estructura temática y de la información en la introducción del ensayo de crítica literaria y la relación con su título me permitieron acercarme, de una manera bastante satisfactoria, al estudio de un aspecto en la dinámica discursiva.

\section{Bibliografía}

Beaugrande, R. A. de y W. V. Dressler. 1986. Introduction to Text Linguistics. Londres/Nueva York: Longman.

Brown, G. y G. Yule. 1983. Discourse Analysis. Londres: Cambridge University Press.

Daneš, F. 1970. "One Instance of Prague School Methodology: Functional Analysis of Utterance and Text". P. Garvin, ed., Method and Theory in Linguistics. The Hague: Mouton. 
Daneš, F. 1974. "Functional Sentence Perspective and the organization of the Text”. F. Daneš, ed., Papers on Functional Sentence Perspective. Praga: Academia.

Grice, H. P. 1975. "Logic and Conversation". P. Cole, ed., Syntax and Semantics3. Speech: Acts. Nueva York.

Halliday, M. A. K. 1982. El lenguaje como semiótica social. La interpretación social del lenguaje y del significado. México: FCE. 1985. An Introduction to Functional Grammar. Londres: E. Arnold. y R. Hasan, 1976. Cohesion in English. Londres: E. Arnold.

Prince, E. P. 1981. "Toward a Taxonomy of Given-New Information". P. Cole, ed., Radical Pragmatics. Nueva York: Academic Press. Citado en G. Brown y G. Yule. 1983.

Quirk, R., S. Greenbaum, G. Leech y J. Svartvik. 1972. A Grammar of Contemporary. English. Londres: Longman.

Rall, M. 1980. "La gramática de dependencia y la enseñanza del alemán como lengua extranjera". Thesis, núm. 6, México.

Rébora, E. 1989. Mensaje e interacción en el ensayo de crítica literaria en la lengua inglesa. Análisis del título y de la introducción. Tesis de Maestría en Lingüística Aplicada. México, UNAM.

Souto, A. 1973. El ensayo. Programa Nacional de Formación de Profesores (ANUIES). Mexico: Complejo Editorial Latinoamericano.

Strawson, P. F. 1950. On Referring en Mind, vol. LIX, Logico-Linguistic Papers. Londres. 